\title{
A comparative pharmacokinetics study of Ashwagandha (Withania somnifera) Root Extract sustained-release capsules: an open-label, randomized, two treatment, two-sequence, two period, single-dose crossover clinical study
}

\author{
${\text { Venkata Krishna Raju Alluri }{ }^{1} \text {, Shefali Thanawala }}^{2 *}$, Vivek Upadhyay ${ }^{3}$
}

\author{
${ }^{1}$ Laila Nutraceuticals, Vijayawada, Andhra Pradesh, India \\ ${ }^{2}$ Inventia Healthcare Ltd., Mumbai, Maharashtra, India \\ ${ }^{3}$ Enem Nostrum Pvt. Ltd., Mumbai, Maharashtra, India
}

Received: 23 November 2021

Accepted: 09 December 2021

\section{*Correspondence:}

Dr. Shefali Thanawala,

Email: shefali.thanawala@inventiahealthcare.com

Copyright: $@$ the author(s), publisher and licensee Medip Academy. This is an open-access article distributed under the terms of the Creative Commons Attribution Non-Commercial License, which permits unrestricted non-commercial use, distribution, and reproduction in any medium, provided the original work is properly cited.

\begin{abstract}
Background: In this open-label, randomized, balanced, two-treatment, two-sequence, two-period, crossover, singledose oral comparative pharmacokinetics study, the pharmacokinetics, safety, and tolerability of test product 'ashwagandha (Withania somnifera)' root extract sustained release capsule $300 \mathrm{mg}$ (Prolanza ${ }^{\mathrm{TM}}$ ), each containing 15 mg withanolides (administered dose: $2 \times 15 \mathrm{mg}$ ) was compared with that of a reference product (organic KSM-66 ashwagandha extract [vegan] capsule, each containing $15 \mathrm{mg}$ withanolides [administered dose: $2 \times 15 \mathrm{mg}$ ]).

Methods: Total 14 healthy men were randomized to receive either the test or the reference product as a single dose of 2 capsules in sequence, administered under fasting conditions. Plasma concentrations of total withanolides, withanolide A and 12-deoxywithastramonolide were measured using validated liquid chromatography-mass spectroscopy/mass spectroscopy.

Results: The test product had higher relative absorption, better relative bioavailability, and longer elimination half-life indicating a sustained-release profile compared to reference. Specifically, the relative bioavailability of the test formulation was 12, 44, and 11 times higher for total withanolides, withanolide A and 12-deoxywithastramonolide, respectively. No adverse events were reported during the study.

Conclusions: The sustained-release profile of the test product, compared to reference product, will provide more longlasting therapeutic effects from a single daily dose (Retrospectively applied on Clinical Trials Registry - India [CTRI]. Application reference number: REF/2020/03/032408). The study reports the unique sustained release formulation of Withania somnifera (Ashwagandha) root extract. The pharmacokinetic study also reports for first time, the successful plasma estimation of withanolide A and 12-deoxywithastraamonolide, the major phytoactives of ashwagandha.
\end{abstract}

Keywords: Withania somnifera, Withanolides, Ashwagandha, Sustained-release, Pharmacokinetic

\section{INTRODUCTION}

Ayurveda is a highly systematized traditional medical system practiced in India since thousands of years. ${ }^{1}$ Root extract of Indian ginseng or Withania somnifera, known as ashwagandha in Sanskrit, is an essential therapeutic used in Ayurvedic medicine. ${ }^{1}$ Ayurveda recommends its use as a Rasayana or "adaptogen" and suggests that it has beneficial effects as a Medhya Rasayana or neuroadaptogen, and antistress, antioxidant, immunomodulatory, rejuvenating, anti-inflammatory, anti-arthritic and antitumor properties., ${ }^{2,3}$ Thus, ashwagandha is used to manage various neuropsychiatric 
and neurodegenerative disorders such as anxiety and stress, Parkinson's disease, Alzheimer's disease, dementia, mood and behavioral disorders, insomnia, etc. It also exerts beneficial effect on diseases like cancer, inflammatory conditions including arthritis, bronchitis, etc., and found effective in gynecological disorders such as meno-metrorrhagia. ${ }^{2-4}$

The principal bioactive compounds of $W$. somnifera are withanolides which can be found in the roots, aerial parts, and berries of the plant. ${ }^{5}$ They are a group of naturally occurring triterpene lactone rings. More than 40 withanolides, nearly 12 alkaloids and several sitoindosides have been isolated and identified from $W$. somnifera. Various alkaloids such as withanine, withananine, pseudowithanine, somniferine, somniferinine, etc. can be isolated from plant parts, though leaves contain alkaloids in the highest concentration. However, for therapeutic purpose and internal administration, Ayurveda recommends use of only the roots and no other plant parts. Chemical constituents of the roots include withanolides A, $\mathrm{D}$, and G; 12-deoxywithastramonolide, withanone; withaferin A; and sitoindosides VII-X. Several studies have evaluated the pharmacological actions of different constituents, mainly, withanolide A and 12deoxywithastramonolide., ${ }^{6,7}$ High performance liquid chromatography techniques to quantify constituents have also been established..$^{5,8}$

Recently, researchers are more inclined towards finding possible uses of $W$. somnifera in treating various disorders. Thus, several trials have assessed the efficacy and safety of W. somnifera for various purposes such as treating stress and anxiety, improving vitality or sexual function, strength training, arthritis treatment, and as an adjuvant to antitubercular, anticancer, or antipsychotic medications. ${ }^{9-20}$ Most of these trials used twice-daily dosages of standard immediate release ashwagandha formulations, which are commonly prescribed in clinical practice. However, till date, the development or use of modified release ashwagandha formulations is not reported.

The advantages of sustained release are improved administrative convenience and dosage compliance. ${ }^{21}$ Based on these, the sponsor of this study developed novel formulation of ashwagandha containing the same dose but in a sustained-release (SR) format for the first time, to provide superior bioavailability of active constituentswithanolides, to exert their therapeutic effect for extended period after administration of a single daily dose.

The disposition and pharmacokinetics of the actives, withanolides of the ashwagandha SR formulation was first evaluated in an in vivo study in male Sprague Dawley rats and compared with marketed immediate release (IR) formulation of ashwagandha (KSM-66). A partially validated LC-MS/MS method was used to simultaneously determine the total withanolides concentration in the formulations. A single oral dose of $50 \mathrm{mg} / \mathrm{kg}$ equivalent withanolides concentration was selected based on the results of exploratory studies. The formulations were well tolerated. The relative bioavailability of total withanolides was found to be higher for SR formulation as compared to IR formulation in terms of exposure, peak plasma concentration $\left(\mathrm{C}_{\max }\right)$ and time to reach maximum concentration $\left(\mathrm{T}_{\max }\right)$. The AUC was found to be higher for SR pellets (AUClast: 69.903 $\pm 35.959 \mathrm{hr} * \mathrm{ng} / \mathrm{ml}$ ) as compared to IR formulation $(26.808 \pm 13.300 \mathrm{hr} * \mathrm{ng} / \mathrm{ml})$. The $\mathrm{C}_{\max }$ of $\mathrm{SR}$ pallets was improved $(12.946 \pm 14.721$ $\mathrm{ng} / \mathrm{ml})$ as compared to IR formulation $(8.920 \pm 5.914$ $\mathrm{ng} / \mathrm{ml}$ ). For $\mathrm{SR}$ formulation the $\mathrm{T}_{\max }$ was found to be 5.46 hours as compared to 0.29 hours of IR formulation. The $\mathrm{T} 1 / 2$ for SR pallets was $11.87 \pm 2.84 \mathrm{hr}$, whereas it was not evaluable for IR formulation. The results confirmed higher relative bioavailability with 2.6-fold increase in exposure of total withanolides for SR formulation as compared to IR formulation.

Based on these results, the pharmacokinetic study in healthy human subjects was designed. The objective of this study was to assess comparative pharmacokinetics of test product (ashwagandha root extract sustained-release capsules $300 \mathrm{mg}$ containing $15 \mathrm{mg}$ withanolides [2x15 mg] of Inventia healthcare Ltd., and Laila nutraceuticals, India; batch No.: MT031) with the reference product (organic KSM-66 ashwagandha extract [vegan] capsules containing $15 \mathrm{mg}$ withanolides [2x15 mg] of Shri Kartikeya Pharma, India; Batch No.: KSM/VG/17/981) in normal healthy adults under fasting conditions. The reference product was used as control and its pharmacokinetic profile was determined to compare with that of the test formulation. We also monitored the safety and tolerability of both products in this population.

\section{METHODS}

\section{Overview of the study design}

This was a randomized, balanced, open-label, twotreatment, two-sequence, two-period, two-way, single-oral dose, crossover, comparative pharmacokinetics study in 14 healthy adult human subjects (Clinical trials registry- India [CTRI] reference number: REF/2020/03/032408). This being the first time ever pharmacokinetic study conducted to determine the estimation of withanolides in human matrix, the sample size of 14 was selected.

\section{Study participants}

In this study, we included healthy adults (age: $18-45$ years, body mass index: $18.50-29.99 \mathrm{~kg} / \mathrm{m}^{2}$ with minimum $45 \mathrm{~kg}$ weight) with normal health status as determined by personal medical and medication history, clinical examination, laboratory examinations and who were willing to use an acceptable and effective method of contraception. Specifically, subjects with clinically acceptable vital parameters (blood pressure, body temperature, pulse rate and respiratory rate), 12-lead electrocardiogram and chest X-ray (posteroanterior view) within last 180 days, and those who tested negative for urine screen for drugs of abuse (including amphetamines, 
barbiturates, benzodiazepines, marijuana, cocaine and morphine) and alcohol breath test were included. All subjects who fulfilled the protocol requirements and provided written consent were included in the study.

Subjects were excluded if they reported hypersensitivity to study product or any of inactive ingredients of test or reference formulation, had clinically significant findings in medical history, physical examination, and laboratory assessments suggestive of specific diseases, or a history of drug/alcohol abuse in the past 1 year or history of smoking. Also, subjects with a history of asthma, urticaria or other significant allergic reactions, intolerance to venipuncture; donated blood or participated in a clinical study involving blood sampling within 90 days prior to study initiation, consumed grapefruit juice (within 72 hours prior dosing) and consumed products with xanthine, tobacco or any alcoholic products within 48 hours prior dosing, etc. were excluded.

\section{Study procedure: clinical phase}

\section{Physician assessment and laboratory tests}

Prior to study initiation, all volunteers provided informed consent in the presence of the investigator or the person designated by the investigator. They were briefed on the procedures and potential risks of participating in the study. The complete study specific informed consent process was carried out under audio visual surveillance and was recorded. The physician confirmed eligibility of the subjects based on their medical history, laboratory investigations, clinical examination (general examination, systemic examination and vital signs). Subjects were instructed not to consume any prescribed medication or any over-the-counter medication including vitamins and herbal products 14 days prior dosing in period- 1 till end of period-2, and no concomitant therapy was administered during the study period.

At the admission day in each period, subjects underwent urine screening for commonly abused substances (amphetamine, benzodiazepines, barbiturates, cocaine, marijuana, and morphine) and breath alcohol test to ensure eligibility for participation.

Physicians assessed safety, well-being, vital signs in the morning before dosing of investigational products and at 3.00, 6.00- and 11.00-hours post dose in each study period and also at the time of discharge in each period. Post-study safety assessment was performed at the end of study which included well-being assessment, clinical examination, hemogram and biochemistry (serum glutamic-oxaloacetic transaminase [SGOT], serum glutamate-pyruvate transaminase [SGPT], bilirubin, creatinine and urea).

\section{Randomization}

Eligible participants were randomized to receive one treatment sequence (test/reference and reference/test sequences, in 1:1 ratio) using a predetermined, computergenerated randomization scheme (PROC PLAN in SAS® [SAS Institute Inc., USA, version 9.4]). Pharmacist dispensing investigational product, principal investigator and the quality assurance personnel were accountable for ensuring compliance to the randomization schedule.

\section{Blinding}

As this was an open-label study, both subjects and investigators were not blinded to the identity of the test or reference product. However, the personnel from the bioanalytical team were completely blinded to the identity of the investigational product administered.

\section{Investigational product administration and other in-house processes}

Subjects remained confined in-house for at least 11 hours pre-dose and 24 hours post-dose in each study period. A single dose (2 capsules, each containing $15 \mathrm{mg}$ withanolides) of test or reference product as per randomization schedule was administered to the subjects in sitting posture with $240 \mathrm{ml}$ potable water at room temperature after at least 10 hours of overnight fasting in period 1. After a washout period of at least 14 days, participants received the other study treatment for period 2. Study personnel instructed subjects to swallow the capsule as a whole and not to chew or crush it. To ensure compliance, a thorough check of the oral cavity using torch and disposable wooden spatula was performed immediately after dosing and the product concentration level in the plasma was evaluated. Duplicate labels of dispensed container were pasted on the 'Dosing' section of individual case report forms. All subjects had identical, standardized meals at check-in night and at 4.00, 8.00- and 12.00-hours post-dose in each study period. Subjects were not allowed to drink water one hour prior to dosing until one-hour post-dose $(240 \mathrm{ml}$ of potable water given during dosing). At all other times, the subjects were allowed to drink water ad libitum. Subjects remained seated or semirecumbent for 2 hours after administration of investigational product, after which they could engage in normal activities while avoiding the severe physical exertion.

\section{Collection and processing of samples}

Blood samples $(5.0 \mathrm{ml})$ were collected in pre-labeled vacutainers containing K2-ethylenediaminetetraacetic acid (EDTA) as the anticoagulant before dosing and at $0.25,0.50,0.75,1.00,2.00,4.00,6.00,8.00,10.00,12.00$, 16.00- and 24.00-hours post-dosing in each period, using an indwelling cannula placed in a forearm vein. The samples were handled in wet ice-water bath and were stored at $-70 \pm 15^{\circ} \mathrm{C}$. Samples were centrifuged at $4^{\circ} \mathrm{C}$ for 10 minutes at $4000 \mathrm{rpm}$ within 60 minutes of last sample collection (short-term excursion permitted up to $8^{\circ} \mathrm{C}$ ), and the plasma was equally transferred into storage vials in 
duplicates (one aliquot for each control and analysis) that were stored at $-70^{\circ} \pm 15^{\circ} \mathrm{C}$ for interim storage.

\section{Bioanalytical phase}

There was no direct measure of efficacy in this study. The selected dose was expected to produce measurable blood levels after administration and the sampling schedule of 13 samples to provide an adequate estimation of $\mathrm{C}_{\max }$ and to cover the plasma concentration-time curve long enough to provide a reliable estimate of the extent of absorption.

The temperature of samples was maintained during transfer and until analysis. The plasma concentrations of Withanolide A, 12-deoxywithastramonolide and total withanolides were estimated using validated liquid chromatography-mass spectrometry/mass spectrometry (LC-MS/MS) methods. Plasma samples were extracted by a solid phase extraction method using Agilent, Bond Elute PLEXA $30 \mathrm{mg}$, 1cc extraction cartridges and methanol as a final elution solvent. The eluent after extraction was evaporated under a stream of nitrogen and the residue was reconstituted with $0.15 \mathrm{~mL}$ of mobile phase before loading into an autosampler. Then, $10 \mu \mathrm{l}$ was injected into the LCMS/MS. All calibration curves and quality control samples were prepared in human plasma by spiking standard solutions. Correlation coefficients for all methods achieved R2 values >0.990. Atorvastatin D5 (BioOrganics, Bengaluru, India) was used as internal standard for withanolide A, 12-deoxywithastramonolide and total withanolides calibration curves were prepared for withanolide A (Laila nutraceuticals, Vijayawada, India) and 12-deoxywithastramonolide (Laila Nutraceuticals, Vijayawada, India).

Analysis of withanolide A, 12-deoxywithastramonolide and total withanolides was carried out using a highperformance liquid chromatography (HPLC) system coupled to a mass detector. The chromatographic separation of all compounds was achieved using Exsil Mono $100 \mathrm{C} 18,3 \mu \mathrm{m},(100 \times 4.6) \mathrm{mm}$ column and mobile phase consisting acetonitrile and $10 \mathrm{mM}$ ammonium formate in water with $0.1 \%$ glacial acetic acid (70:30) at a flow rate of $0.5 \mathrm{ml} / \mathrm{min}$. All analytes were detected using a mass detector equipped with turbo ion spray ionization source in a positive ionization mode. The analytes were detected in the multiple reaction monitoring mode. The mass spectrometer transitions (precursor/product) were as follows: withanolide A (488.500/263.200), 12deoxywithastramonolide (471.400/263.200) and atorvastatin D5 (564.400/445.400). The optimized parameters for the ionization source included spray voltage set at $5.5 \mathrm{kV}$, nebulizer gas flow rate of 40 units, turbo gas flow rate of 70 units, curtain gas flow rate of 35 units, and temperature of turbo gas set at $600^{\circ} \mathrm{C}$. The collision energies applied depended on the fragmentation behavior of the molecule investigated. Using the optimized method, the obtained limits of quantification (LOQ) were as follows: Withanolide A, $0.025 \mathrm{ng} / \mathrm{ml}$ and 12deoxywithastramonolide, $0.100 \mathrm{ng} / \mathrm{ml}$. Each calibration curve was analyzed individually by using least square weighted linear regression (1/x2). Inter-day and intraday variability for each analyte were within the acceptance criteria.

\section{Pharmacokinetic and statistical analysis}

The pharmacokinetic dataset comprised of the estimates of $\mathrm{C}_{\max }$ and area under curve (AUC) parameters for two periods. We used the estimated plasma concentration vs. time profiles of the actives, and calculated the primary variables that included $\mathrm{C}_{\max }, \mathrm{AUC}_{0-\mathrm{t}}$ and $\mathrm{AUC}_{0-\infty}$ and secondary variables that included $\mathrm{T}_{\max }$, AUC_\% Extrap_obs, $\mathrm{t}_{1 / 2}$ and $\mathrm{K}_{\mathrm{el}}$. Summary statistics and statistical analysis were performed only on those subjects for whom the pharmacokinetic parameters were estimable. All concentration values below the limit of quantification were set to "zero" and missing concentrations were reported and not included for analysis.

Analysis of variance (ANOVA) was used as the statistical test and calculated least square means for test and reference formulations, intra-subject variability and power for $\ln$-transformed pharmacokinetic parameters $\mathrm{C}_{\max }$, $\mathrm{AUC}_{0-\mathrm{t}}$ and $\mathrm{AUC}_{0-\infty}$. The ANOVA model included fixed effects of sequence, treatment, period and subject (sequence). Sequence effects were tested using the subject (sequence) effect as the error term. All main effects were tested at the 0.05 level of significance. The geometric least square means ratio [T/R] was used with respect to the pharmacokinetic parameter $\mathrm{AUC}_{0-\mathrm{t}}$ for total withanolides, withanolide A, and 12-Deoxywithastramonolide to assess the relative bioavailability of the test product as compared to the reference formulation. The geometric least square means of test and reference formulations, its ratio, $90 \%$ confidence interval (CI) for geometric least square mean ratio T/R and 2 one-sided tests for $90 \%$ CI limits was calculated for pharmacokinetic parameters $\mathrm{C}_{\max }, \mathrm{AUC}_{0-\mathrm{t}}$ and $\mathrm{AUC}_{0-\infty}$. All statistical analyses were performed using SAS® software (version 9.4, SAS institute inc, Cary, NC).

\section{Safety analysis}

Study personnel monitored the subjects for any adverse events and serious adverse events throughout the study. Safety evaluation was done on the basis of outcomes of vital signs and physical examination (general and systemic) during each study period and clinical laboratory assessments performed during post study safety assessment (at the time of collection of last PK sample in last study period).

\section{Ethical considerations}

The study was conducted in compliance with Indian Council of Medical Research (ICMR) guidelines for biomedical research on human subjects; the International Council for Harmonization of technical requirements for pharmaceuticals for human use (Step 5) E6 (R2) guidance on good clinical practice (ICH-GCP); Declaration of Helsinki (Brazil, October 2013); procedures oriented to GLP, OECD (Organization for economic co-operation and 
development); and schedule L-I of D and C rules 1945, new drug and clinical trial rule 2019 (Central drugs standard control organization [CDSCO] guidelines, Good Clinical Practice for clinical research in India). An independent ethics committee (The Ethos ethics committee) approved the study. All subjects provided written informed consent voluntarily before their enrollment in this study. All study procedures complied with the approved study protocol and there were no changes in the conduct of the study except one planned deviation in sample handling and processing.

\section{RESULTS}

\section{Study flow}

Study flow was summarized in Figure 1. Sixteen $(14+2$ standby subjects up to dosing in period-1) healthy willing adult male human subjects were enrolled, and 14 subjects were randomized and dosed in both periods during the period $22^{\text {nd }}$ January 2020 (Check-in of period 01) to $07^{\text {th }}$ February 2020 (Post study safety assessment). There were no dropouts in either period and all participants completed the study. The data were analyzed both biologically and statistically for all 14 participants. Thus, samples collected from 14 subjects were analyzed and pharmacokinetic concentrations were utilized for pharmacokinetic analysis.

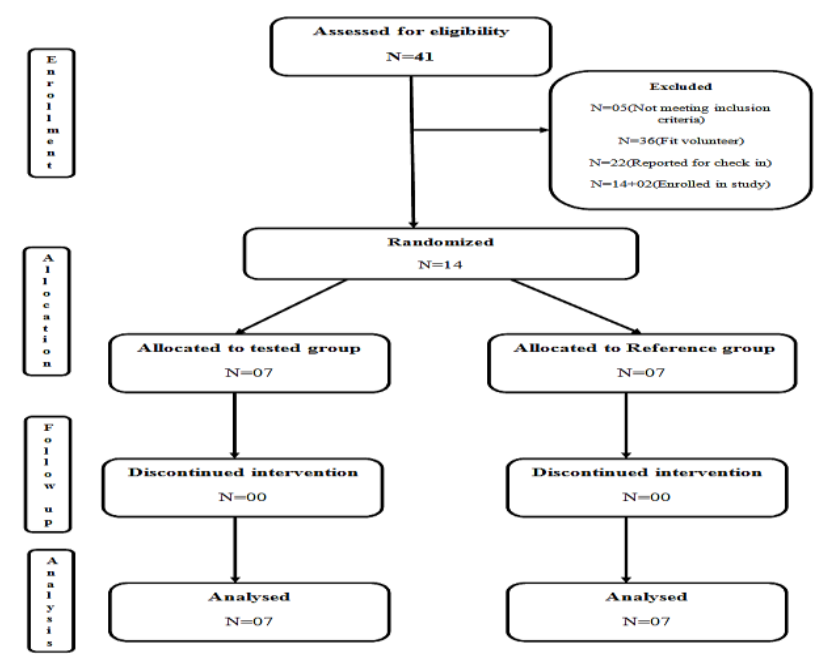

Figure 1: Study flowchart.

\section{Demographic and baseline characteristics}

Subjects were aged 23-42 years and had weight (range: $53.89-80.64 \mathrm{~kg})$, height $(156.0-176.0 \mathrm{~cm})$ and body mass indices (range: 18.98 to $29.26 \mathrm{~kg} / \mathrm{m}^{2}$ ) within clinically acceptable normal range (Table 1). All the subjects were healthy as confirmed by the physician assessments.

Table 1: Baseline demographic details.

\begin{tabular}{|ll|}
\hline Parameters & Value $($ mean \pm SD $)$ \\
\hline Age $($ years $)$ & $35.64 \pm 5.40$ \\
\hline BMI $\left(\mathbf{K g} / \mathbf{m}^{\mathbf{2}}\right)$ & $23.66 \pm 2.83$ \\
\hline
\end{tabular}

\section{Pharmacokinetic results}

Linear mean plasma concentration vs. time curves for total withanolides, withanolide A, and 12Deoxywithastramonolide showed consistently higher plasma concentrations for test product vs. reference product between 1-to-24-hour post-dose (Figure 2). Tables 2-3 present the pharmacokinetic parameters estimated for test vs. reference product. $90 \%$ CI of geometric least squares mean ratio (GLSMR) (test vs. reference) for total withanolides were $416.20 \%-596.90 \%$ for $\mathrm{C}_{\max }, 934.82 \%-$ $1467.92 \%$ for $\mathrm{AUC}_{0-\mathrm{t}}$, and $792.89 \%-1088.56 \%$ for $\mathrm{AUC}_{0-}$ $\infty$. The corresponding values for withanolide A were 408.47\%-721.40\% for $\mathrm{C}_{\max }$ and $2895.27 \%-6725.10 \%$ for $\mathrm{AUC}_{0-\mathrm{t}}$. For 12-deoxywithastramonolide, the $90 \% \mathrm{CI}$ of GLSMR (test vs. reference) were 388.58\%-542.86\% for $\mathrm{C}_{\max }, 862.21 \%-1319.57 \%$ for $\mathrm{AUC}_{0-\mathrm{t}}$, and $741.92 \%-$ $997.01 \%$ for $\mathrm{AUC}_{0-\infty}$. The relative bioavailability for test formulation was 12 times superior for total withanolides, 44 times superior for withanolide $\mathrm{A}$, and 11 times superior for 12-Deoxywithastramonolide compared to the reference formulation. The test product when compared with the reference product had higher relative absorption of total withanolides (mean \pm standard deviation [SD]: $\mathrm{C}_{\max }$ $(\mathrm{ng} / \mathrm{ml}), 3.0701 \pm 1.26170$ vs. $0.6480 \pm 0.35187 ; \mathrm{AUC}_{0-\mathrm{t}}$ (hr*ng/ml), $24.4951 \pm 10.16071$ vs. $2.2760 \pm 1.16395)$, and longer elimination $\mathrm{t}_{1 / 2}(\mathrm{hr})($ mean $\pm \mathrm{SD}, 7.0708 \pm 2.00660 \mathrm{vs}$. $2.2789 \pm 0.44337)$.
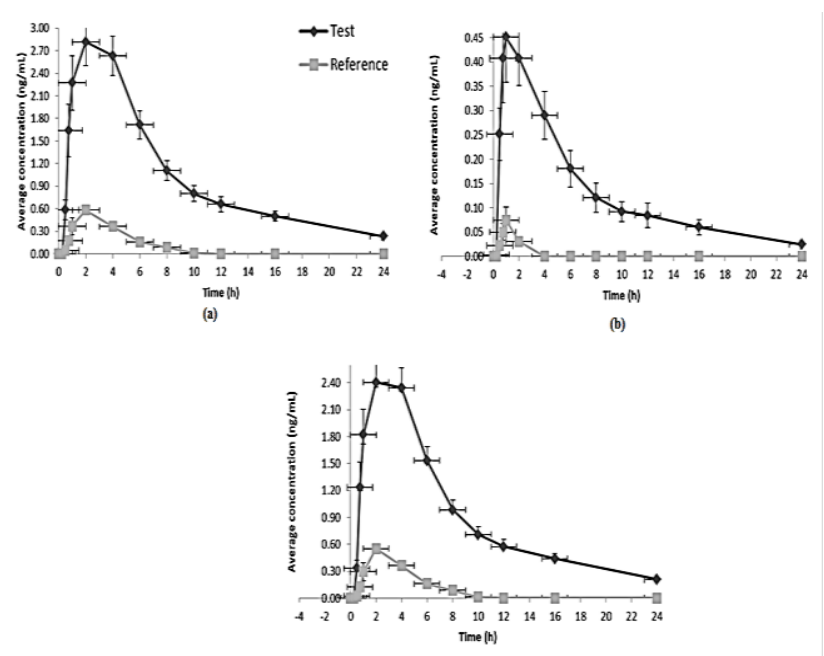

(c)

Figure 2: Plasma concentration (mean \pm SD) of test product vs. reference product between 1-to-24-hour post-dose. (a) Average concentration (ng/ml) for total withanolides; (b) average concentration $(\mathrm{ng} / \mathrm{ml})$ for withanolide A; (c) average concentration $(\mathrm{ng} / \mathrm{ml})$ for 12-Deoxywithastramonolide.

\section{Safety results}

The laboratory results showed no abnormal changes, and no clinically significant or serious adverse events were reported for both groups during the study and the poststudy assessments. 
Table 2: Pharmacokinetic parameters of total withanolides after a single oral dose of test vs reference product in healthy volunteers.

\begin{tabular}{|c|c|c|c|}
\hline PK Parameter & Test $^{\dagger}$ & Reference & GLSMR $^{\S}(90 \%$ CI) \\
\hline \multicolumn{4}{|l|}{$\mathrm{C}_{\max }(\mathrm{ng} / \mathrm{ml})$} \\
\hline $\begin{array}{l}\text { Arithmetic Mean } \pm \text { SD } \\
(\% \mathrm{CV})\end{array}$ & $\begin{array}{l}3.0701 \pm 1.26170 \\
(41.10)\end{array}$ & $\begin{array}{l}0.6480 \pm 0.35187 \\
(54.30)\end{array}$ & \multirow{2}{*}{$\begin{array}{l}498.43 \\
(416.20-596.90)\end{array}$} \\
\hline Geometric least square mean & 2.8326 & 0.5683 & \\
\hline \multicolumn{4}{|l|}{$\operatorname{AUC}_{0-\mathrm{t}}(\mathrm{hr} * \mathrm{ng} / \mathrm{ml})$} \\
\hline $\begin{array}{l}\text { Arithmetic Mean } \pm \text { SD } \\
(\% \mathrm{CV})\end{array}$ & $\begin{array}{l}24.4951 \pm 10.16071 \\
(41.48)\end{array}$ & $\begin{array}{l}2.2760 \pm 1.16395 \\
(51.14)\end{array}$ & \multirow{2}{*}{$\begin{array}{l}1171.43 \\
(934.82-1467.92)\end{array}$} \\
\hline Geometric least square mean & 22.6014 & 1.9294 & \\
\hline \multicolumn{4}{|l|}{$\mathbf{A U C}_{0-\infty},(\mathbf{h r} * \mathbf{n g} / \mathrm{ml})$} \\
\hline $\begin{array}{l}\text { Arithmetic Mean } \pm \text { SD } \\
(\% \mathrm{CV})\end{array}$ & $\begin{array}{l}27.3474 \pm 11.34489 \\
(41.48)\end{array}$ & $\begin{array}{l}3.1117 \pm 0.95045 \\
(30.54)\end{array}$ & \multirow[t]{2}{*}{$929.04(792.89-1088.56)$} \\
\hline Geometric least square mean & 27.8972 & 3.0028 & \\
\hline \multicolumn{4}{|l|}{ AUC_\% Extrap_obs } \\
\hline $\begin{array}{l}\text { Arithmetic Mean } \pm \text { SD } \\
(\% \mathrm{CV})\end{array}$ & $\begin{array}{l}10.2456 \pm 4.72137 \\
(46.08)\end{array}$ & $\begin{array}{l}14.3537 \pm 7.78950 \\
(54.27)\end{array}$ & \\
\hline \multicolumn{4}{|l|}{$\mathbf{T}_{\max }(\mathbf{h r})^{\#}$} \\
\hline $\begin{array}{l}\text { Median } \\
(\min -\max )\end{array}$ & $\begin{array}{l}2.0000 \\
(0.750-4.000)\end{array}$ & $\begin{array}{l}2.0000 \\
(1.000-4.000)\end{array}$ & \\
\hline \multicolumn{4}{|l|}{$t_{1 / 2}(h r)$} \\
\hline $\begin{array}{l}\text { Arithmetic Mean } \pm \text { SD } \\
(\% \mathrm{CV})\end{array}$ & $\begin{array}{l}7.0708 \pm 2.00660 \\
(28.38)\end{array}$ & $\begin{array}{l}2.2789 \pm 0.44337 \\
(19.46)\end{array}$ & \\
\hline \multicolumn{4}{|l|}{$\mathrm{K}_{\mathrm{el}}(\mathbf{1} / \mathbf{h r})$} \\
\hline $\begin{array}{l}\text { Arithmetic Mean } \pm \text { SD } \\
(\% \mathrm{CV})\end{array}$ & $\begin{array}{l}0.1048 \pm 0.02643 \\
(25.21)\end{array}$ & $\begin{array}{l}0.3133 \pm 0.05216 \\
(16.65)\end{array}$ & \\
\hline
\end{tabular}

AUC, area under the curve; $\mathrm{AUC}_{0-\infty}, \mathrm{AUC}$ from time zero to infinity; $\mathrm{AUC}_{0-\mathrm{t}}$, AUC from time zero to the time of the last measurable analyte concentration; $\mathrm{C}_{\max }$, maximum plasma analyte concentration; CI, confidence interval; Extrap_obs, extrapolated observation; GLSMR, geometric least squares mean ratio; $K_{\mathrm{el}}$, elimination Rate Constant; $\mathrm{T}_{\max }$, time to reach peak plasma concentration; $\mathrm{t}_{1 / 2}$, plasma elimination half-life. ${ }^{\dagger}$ Ashwagandha root extract sustained-release (SR) capsules $300 \mathrm{mg}$ (containing $15 \mathrm{mg}$ withanolides) $(2 \mathrm{x} 15 \mathrm{mg}$ ). † Organic Ashwagandha extract (Vegan) capsules (containing $15 \mathrm{mg}$ withanolides) (2 x $15 \mathrm{mg}) .{ }^{\S}$ Ratio was calculated as Test/Reference.

Table 3: Pharmacokinetic parameters of withanolide A after a single oral dose of test vs reference product in healthy volunteers.

\begin{tabular}{|c|c|c|c|}
\hline PK Parameter & Test $^{\dagger}$ & Reference & GLSMR $^{\S}(90 \%$ CI) \\
\hline \multicolumn{4}{|l|}{$\mathrm{C}_{\max }(\mathrm{ng} / \mathrm{ml})$} \\
\hline $\begin{array}{l}\text { Arithmetic Mean } \pm \text { SD } \\
(\% \mathrm{CV})\end{array}$ & $\begin{array}{l}0.4914 \pm 0.34137 \\
(69.48)\end{array}$ & $\begin{array}{l}0.0855 \pm 0.10025 \\
(117.25)\end{array}$ & \multirow{2}{*}{$\begin{array}{l}542.84 \\
(408.47-721.40)\end{array}$} \\
\hline Geometric least square mean & 0.4261 & 0.0785 & \\
\hline \multicolumn{4}{|l|}{$\operatorname{AUC}_{0-\mathrm{t}}(\mathrm{hr} * \mathrm{ng} / \mathrm{ml})$} \\
\hline $\begin{array}{l}\text { Arithmetic Mean } \pm \text { SD } \\
(\% \mathrm{CV})\end{array}$ & $\begin{array}{l}3.0566 \pm 2.39626 \\
(78.40)\end{array}$ & $\begin{array}{l}0.0929 \pm 0.09199 \\
(98.99)\end{array}$ & \multirow{2}{*}{$\begin{array}{l}4412.60 \\
(2895.27-6725.10)\end{array}$} \\
\hline Geometric least square mean & 2.8276 & 0.0641 & \\
\hline \multicolumn{4}{|l|}{$\operatorname{AUC}_{0-\infty}\left(h^{*}{ }^{*} \mathrm{ng} / \mathrm{ml}\right)$} \\
\hline $\begin{array}{l}\text { Arithmetic Mean (SD) } \\
(\% \mathrm{CV})\end{array}$ & $\begin{array}{l}3.5783 \pm 2.53040 \\
(70.71)\end{array}$ & $\begin{array}{l}0.1211 \pm \mathrm{NE} \\
(\mathrm{NE})\end{array}$ & \multirow[t]{2}{*}{ NE } \\
\hline Geometric least square mean ${ }^{¥}$ & $\mathrm{NE}$ & NE & \\
\hline \multicolumn{4}{|l|}{ AUC_\% Extrap_obs } \\
\hline $\begin{array}{l}\text { Arithmetic Mean } \pm \text { SD } \\
(\% \mathrm{CV})\end{array}$ & $\begin{array}{l}15.1823 \pm 12.19587 \\
(80.33)\end{array}$ & $\begin{array}{l}22.0605 \pm \mathrm{NE} \\
(\mathrm{NE})\end{array}$ & \\
\hline \multicolumn{4}{|l|}{$\mathbf{T}_{\max }(\mathbf{h r})^{\#}$} \\
\hline Median (min-max) & $1.0000(0.500-2.000)$ & $1.0000(0.000-2.000)$ & \\
\hline \multicolumn{4}{|l|}{$\mathbf{t}_{1 / 2}(\mathbf{h r})$} \\
\hline $\begin{array}{l}\text { Arithmetic Mean } \pm \text { SD } \\
(\% \mathrm{CV})\end{array}$ & $\begin{array}{l}7.4561 \pm 5.91665 \\
(79.35)\end{array}$ & $\begin{array}{l}0.7406 \pm \mathrm{NE} \\
(\mathrm{NE})\end{array}$ & \\
\hline \multicolumn{4}{|l|}{$K_{\text {el }}(1 / h r)$} \\
\hline $\begin{array}{l}\text { Arithmetic Mean } \pm \text { SD } \\
(\% \mathrm{CV})\end{array}$ & $\begin{array}{l}0.1219 \pm 0.05192 \\
(42.61)\end{array}$ & $\begin{array}{l}0.9359 \pm \mathrm{NE} \\
(\mathrm{NE})\end{array}$ & \\
\hline \multicolumn{4}{|c|}{ 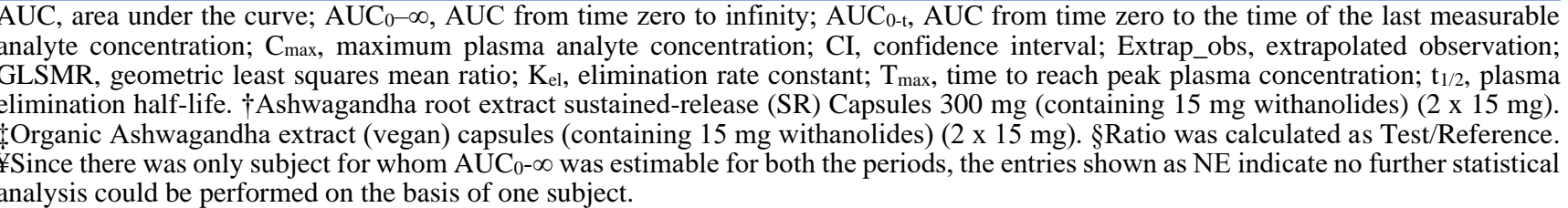 } \\
\hline
\end{tabular}


Table 4. Pharmacokinetic parameters of 12-deoxywithastramonolide after a single oral dose of test versus reference product in healthy volunteers.

\begin{tabular}{|c|c|c|c|}
\hline Pharmacokinetic Parameter & Test $^{\dagger}$ & Reference & GLSMR $^{\S}(90 \%$ CI $)$ \\
\hline \multicolumn{4}{|l|}{$C_{\max }(n g / m l)$} \\
\hline $\begin{array}{l}\text { Arithmetic mean } \pm \text { SD } \\
(\% \mathrm{CV})\end{array}$ & $\begin{array}{l}2.6673 \pm 1.03710 \\
(38.88)\end{array}$ & $0.6067 \pm 0.31355(51.68)$ & \multirow{2}{*}{$\begin{array}{l}459.29(388.58- \\
542.86)\end{array}$} \\
\hline Geometric least square mean & 2.4739 & 0.5387 & \\
\hline \multicolumn{4}{|l|}{$\mathrm{AUC}_{0-\mathrm{t}}(\mathrm{h} \times \mathrm{ng} / \mathrm{ml})$} \\
\hline $\begin{array}{l}\text { Arithmetic mean } \pm \text { SD } \\
(\% \mathrm{CV})\end{array}$ & $\begin{array}{l}21.2996 \pm 8.32103 \\
(39.07)\end{array}$ & $2.1650 \pm 1.08822(50.26)$ & \multirow{2}{*}{$\begin{array}{l}1066.65(862.21- \\
1319.57)\end{array}$} \\
\hline Geometric least square mean & 19.7644 & 1.8529 & \\
\hline \multicolumn{4}{|l|}{$\mathbf{A U C}_{0-\infty}(\mathrm{h} \times \mathrm{ng} / \mathrm{ml})$} \\
\hline $\begin{array}{l}\text { Arithmetic mean } \pm \text { SD } \\
(\% \mathrm{CV})\end{array}$ & $\begin{array}{l}24.1586 \pm 9.42073 \\
(39.00)\end{array}$ & $\begin{array}{l}2.9720 \pm 0.89782 \\
(30.21)\end{array}$ & \multirow{2}{*}{$\begin{array}{l}860.06(741.92- \\
997.01)\end{array}$} \\
\hline Geometric least square mean & 24.6859 & 2.8703 & \\
\hline \multicolumn{4}{|l|}{ AUC_\% Extrap_obs, } \\
\hline $\begin{array}{l}\text { Arithmetic mean } \pm \text { SD } \\
(\% \mathrm{CV})\end{array}$ & $\begin{array}{l}11.7730 \pm 4.91930 \\
(41.78)\end{array}$ & $\begin{array}{l}15.0124 \pm 7.82010 \\
(52.09)\end{array}$ & \\
\hline \multicolumn{4}{|l|}{$\mathbf{T}_{\max }(\mathbf{h})^{\#}$} \\
\hline $\begin{array}{l}\text { Median } \\
(\min -\max )\end{array}$ & $\begin{array}{l}2.0000 \\
(2.000-4.020)\end{array}$ & $\begin{array}{l}2.0000 \\
(1.000-4.000)\end{array}$ & \\
\hline \multicolumn{4}{|l|}{$\mathbf{t}_{1 / 2}(\mathbf{h})$} \\
\hline $\begin{array}{l}\text { Arithmetic mean } \pm \text { SD } \\
(\% \mathrm{CV})\end{array}$ & $\begin{array}{l}7.5317 \pm 2.66857 \\
(35.43)\end{array}$ & $\begin{array}{l}2.2909 \pm 0.43322 \\
(18.91)\end{array}$ & \\
\hline \multicolumn{4}{|l|}{$K_{\text {el }}(1 / h)$} \\
\hline $\begin{array}{l}\text { Arithmetic mean } \pm \text { SD } \\
(\% \mathrm{CV})\end{array}$ & $\begin{array}{l}0.1025 \pm 0.03455 \\
(33.72)\end{array}$ & $\begin{array}{l}0.3110 \pm 0.04980 \\
(16.01)\end{array}$ & \\
\hline
\end{tabular}

Legend: $\mathrm{AUC}$, area under the curve; $\mathrm{AUC}_{0-\infty}$, $\mathrm{AUC}$ from time zero to infinity; $\mathrm{AUC}_{0-\mathrm{t}}$, $\mathrm{AUC}$ from time zero to the time of the last measurable analyte concentration; $\mathrm{C}_{\max }$, maximum plasma analyte concentration; CI, confidence interval; Extrap_obs, extrapolated observation; GLSMR, geometric least square mean ratio; $\mathrm{K}_{\mathrm{el}}$, elimination rate constant; $\mathrm{T}_{\max }$, time to reach peak plasma concentration; $\mathrm{t}_{1 / 2}$, plasma elimination half-life. ${ }^{\dagger}$ Ashwagandha root extract sustained-release (SR) capsule $300 \mathrm{mg}$ (containing $15 \mathrm{mg}$ withanolides) (2x15 $\mathrm{mg})$. ${ }^{*}$ Organic Ashwagandha extract (vegan) capsule (containing $15 \mathrm{mg}$ withanolides) $(2 \times 15 \mathrm{mg})$. ${ }^{\S}$ Ratio was calculated as test:reference.

\section{DISCUSSION}

The objective of this study was to assess the pharmacokinetics and safety of the test formulation as compared to the reference formulation in normal healthy adult human subjects under fasting conditions.

Study design was adequate for determining pharmacokinetic parameters of both test and reference formulations. A washout period of 14 days between each period minimized the carryover effects and ensured complete elimination of the investigational product from the body.

Higher plasma concentrations of total withanolides, withanolide A, and 12-deoxywithastramonolide reported for test product demonstrate the enhanced pharmacological effects of the ashwagandha root extract sustained-release capsules than the reference product. The relative bioavailability for test product was significantly higher, i.e., 12, 44, and 11 times for total withanolides, withanolide A, and 12-deoxywithastramonolide; respectively, as compared to the reference product. The test product when compared with the reference product had higher relative absorption for total withanolides (mean \pm SD: $\quad \mathrm{C}_{\max } \quad(\mathrm{ng} / \mathrm{ml}), \quad 3.0701 \pm 1.26170 \quad$ vs. $0.6480 \pm 0.35187 ; \mathrm{AUC}_{0-\mathrm{t}}(\mathrm{hr} * \mathrm{ng} / \mathrm{ml}), 24.4951 \pm 10.16071$ vs. 2.2760 \pm 1.16395$)$. Being a sustained-release formulation, as expected, the test product exhibited longer elimination half-life (mean \pm SD, 7.0708 \pm 2.00660 vs. $2.2789 \pm 0.44337 \mathrm{hr}$ ) for total withanolides as compared to the reference product. The safety and tolerability of the test product was evident with the absence of adverse events recorded during the study and post-study safety assessment.

Our study is a first-in-human study providing detailed pharmacokinetic data on two of the several reported pharmacological actives present in ashwagandha (Withania somnifera) root, namely, withanolide A, and 12deoxywithastramonolide as well as total withanolides. Most researchers have focused on pharmacological activity of withanolides, such as antitumor, antiinflammatory, cognition promoting, anxiolytic, 'gammaaminobutyric acid'-mimetic effects. ${ }^{3,22}$ Few pharmacokinetic studies on ashwagandha are reported to be conducted in animal models such as mice, guinea pigs, and buffalo calves. ${ }^{23-27}$ There are no human studies providing detailed data on pharmacokinetics of ashwagandha root extract or bioactive compounds therein. Only one human study with 13 enrolled patients with advanced stage high-grade osteosarcoma reported conducting pharmacokinetic analysis of withaferin-A, though there were no detectable levels of withaferin-A in 
any of the samples collected. ${ }^{28}$ Our study adds to the growing literature on ashwagandha by providing the much-needed data on pharmacokinetics of these bioactive compounds and filling these gaps in knowledge. Rigorous methodology, quality control, and completely analyzable data from all enrolled participants add to the strength of this study. A novel feature of this study is the use of sustained release formulation of ashwagandha root extract. Research has demonstrated that medicines which need to be taken over prolonged duration have a better compliance if they need to be administered with lesser frequency. ${ }^{29}$ Thus, it is expected that a single daily dose of ashwagandha SR capsule will improve the subject compliance for ashwagandha, as it is often administered for chronic conditions and in multiple daily doses. Also, as demonstrated by the pharmacokinetic analysis, the sustained release formulation will likely maintain a reasonably constant therapeutic level of the active ingredients in the body, thereby improving the efficacy of the product. However, it must be noted that all the study participants were healthy young males and the sample size was relatively small. Also, only two of the several reported pharmacological actives for Ashwagandha were evaluated in this study. Thus, further research is required to evaluate the pharmacokinetics of more bio-actives from this promising herbal ingredient.

\section{CONCLUSION}

In this group of healthy Indian men, the test productashwagandha SR capsules when compared with reference product, reported higher relative absorption, superior relative bioavailability, and longer elimination half-life for analytes withanolide $\mathrm{A}$, and 12-deoxywithastramonolide and total withanolides. Both test and reference products were safe and well-tolerated by the subjects.

\section{ACKNOWLEDGMENTS}

Author would like to thank all the study participants who completed study per protocol. Publication of these results should not be considered as an endorsement of any product used in this study by any of the organizations or universities where authors are affiliated. Authors would also thanks to Dr. Vidula Bhole and Parth Tiwari from CBCC global research to provide medical writing support.

Funding: Funding sources by Laila Nutraceuticals, Vijayawada, Andhra Pradesh and Inventia Healthcare Ltd., Mumbai, Maharashtra.

Conflict of interest: None declared

Ethical approval: The study was approved by the Institutional Ethics Committee (Registration no.: ECR/139/Indt/MH/2013/RR-16), CTRI reference no. of the trial: REF/2020/03/032408).

\section{REFERENCES}

1. Central Council for Research in Ayurveda and Siddha; Government of India; Department of AYUSH. Ashwagandha, In the Ayurvedic pharmacopoeia of India, AYUSH: New Delhi, India. 2008;1(1):19-20.

2. Balasubramani SP, Venkatasubramanian $P$, Kukkupuni SK, Patwardhan B. Plant-based Rasayana drugs from Ayurveda. Chin J Integr Med. 2011;17(2):88-94.

3. Singh N, Bhalla M, De Jager P, Gilca M. An overview on ashwagandha: A Rasayana (Rejuvenator) of Ayurveda. Afr J Tradit Complement Altern Med. 2011;8(5S):208-13.

4. Verma SK, Kumar A. Therapeutic uses of Withania somnifera (ashwagandha) with a note on withanolides and its pharmacological actions. Asian J Pharm Clin Res. 2011;4(1):1-4.

5. Mirjalili MH, Moyano E, Bonfill M, Cusido RM, Palazón J. Steroidal lactones from Withania somnifera, an ancient plant for novel medicine. Molecules. 2009; 14(7):2373-93.

6. Girme A, Saste G, Pawar S, Balasubramaniam AK, Musande K, Darji B, et al. Investigating 11 withanosides and Withanolides by UHPLC-PDA and Mass Fragmentation Studies from Ashwagandha (Withania somnifera). ACS omega. 2020;5(43):27933-43.

7. Tohda C, Kuboyama T, Komatsu K. Search for natural products related to regeneration of the neuronal network. Neurosignals, 2005;14(1-2):34-45.

8. Dhanani T, Shah S, Gajbhiye N, Kumar S. Effect of extraction methods on yield, phytochemical constituents and antioxidant activity of Withania somnifera. Arab J Chem. 2017;10:S1193-9.

9. Chandrasekhar K, Kapoor J, Anishetty S. A prospective, randomized double-blind, placebocontrolled study of safety and efficacy of a highconcentration full-spectrum extract of ashwagandha root in reducing stress and anxiety in adults. Indian $\mathbf{J}$ Psychol Med. 2012;34(3):255-62.

10. Choudhary D, Bhattacharyya S, Bose S. Efficacy and safety of Ashwagandha (Withania somnifera (L.) Dunal) root extract in improving memory and cognitive functions. J Diet Suppl. 2017;14(6):599-612.

11. Pingali U, Pilli R, Fatima N. Effect of standardized aqueous extract of Withania somnifera on tests of cognitive and psychomotor performance in healthy human participants. Pharmacogn Res. 2014;6(1):12-8.

12. Pratte MA, Nanavati KB, Young V, Morley CP. An alternative treatment for anxiety: a systematic review of human trial results reported for the Ayurvedic herb ashwagandha (Withania somnifera). J Altern Complement Med. 2014;20(12):901-8.

13. Lopresti AL, Drummond PD, Smith SJ. A randomized, double-blind, placebo-controlled, crossover study examining the hormonal and vitality effects of ashwagandha (Withania somnifera) in aging, overweight males. Am J Mens Health. 2019;13(2):1557988319835985.

14. Dongre S, Langade D, Bhattacharyya S. Efficacy and safety of Ashwagandha (Withania somnifera) root 
extract in improving sexual function in women: a pilot study. Biomed Res Int. 2015;284154:1-9.

15. Mamidi P, Thakar A. Efficacy of Ashwagandha (Withania somnifera Dunal. Linn.) in the management of psychogenic erectile dysfunction. Ayu. 2011;32(3):322-8.

16. Ziegenfuss TN, Kedia AW, Sandrock JE, Raub BJ, Kerksick CM, Lopez HL. Effects of an aqueous extract of Withania somnifera on strength training adaptations and recovery: The STAR Trial. Nutrients. 2018;10(11):1807.

17. Kumar G, Srivastava A, Sharma SK, Rao TD, Gupta YK. Efficacy safety evaluation of Ayurvedic treatment (Ashwagandha powder and Sidh Makardhwaj) in rheumatoid arthritis patients: a pilot prospective study. Indian J Med. Res. 2015;141(1):100-6.

18. Kumar R, Rai J, Kajal N, Devi P. Comparative study of effect of Withania somnifera as an adjuvant to DOTS in patients of newly diagnosed sputum smear positive pulmonary tuberculosis. Indian $\mathrm{J}$ Tuberc. 2018;65(3):246-51.

19. Biswal BM, Sulaiman SA, Ismail HC, Zakaria H, Musa KI. Effect of Withania somnifera (Ashwagandha) on the development of chemotherapy-induced fatigue and quality of life in breast cancer patients. Integr Cancer Ther. 2013;12(4):312-22.

20. Chengappa KR, Brar JS, Gannon JM, Schlicht PJ. Adjunctive use of a standardized extract of Withania somnifera (Ashwagandha) to treat symptom exacerbation in schizophrenia: a randomized, doubleblind, placebo-controlled study. J Clin Psychiatry. 2018;79(5):17m11826.

21. Chen Z, Zhu Q, Qi J, Lu Y, Wu W. Sustained and controlled release of herbal medicines: The concept of synchronized release. Int J Pharm. 2019;560:116-25.

22. Wei Z, Li T, Su H, Wang Q, Kuang H. Pharmacological Effects of Withanolides. Biomed J Sci Tech Res. 2020;25(3):19243-8.

23. Patil D, Gautam M, Mishra S, Karupothula S, Gairola S, Jadhav S et al. Determination of withaferin A and withanolide A in mice plasma using highperformance liquid chromatography-tandem mass spectrometry: application to pharmacokinetics after oral administration of Withania somnifera aqueous extract. J Pharm Biomed Anal. 2013;80:203-12.

24. Dai T, Jiang W, Guo Z, Wang Z, Huang M, Zhong G et al. Studies on oral bioavailability and first-pass metabolism of withaferin A in rats using LC-MS/MS and Q-TRAP. Biomed Chromatogr. 2019;33(9):e4573.

25. Wang F, Zhao J, Bai J, Gao K, Cui D et al. Liquid chromatography-tandem mass spectrometry to assess pharmacokinetics and tissue distribution of withaferin A in rats. J Chromatogr A. 2019;1122:90-5.

26. Yang L, Meng X, Kuang H. Comparisons of the pharmacokinetic and tissue distribution profiles of withanolide $\mathrm{B}$ after intragastric administration of the effective part of Datura metel L. in normal and psoriasis guinea pigs. J Chromatogr A. 2018;1083:284-8.

27. Dahikar PR, Kumar N, Sahni Y. Pharmacokinetics of Withania somnifera (Ashwagandha) in healthy buffalo calves. Buffalo Bull. 2012;31(4):219-23.

28. Pires N, Gota V, Gulia A, Hingorani L, Agarwal M, Puri A. Safety and Pharmacokinetics of Withaferin-A in advanced stage high grade Osteosarcoma: A phase I trial. J Ayurveda Integr Med. 2020;11(1):68-72.

29. Laufs U, Rettig-Ewen V, Böhm M. Strategies to improve drug adherence. Eur Heart J. 2011;32(3):264-8.

Cite this article as: Alluri VKR, Thanawala S, Upadhyay V. A comparative pharmacokinetics study of Ashwagandha (Withania somnifera) Root Extract sustained-release capsules: an open-label, randomized, two treatment, two-sequence, two period, single-dose crossover clinical study. Int J Basic Clin Pharmacol 2022;11:26-34. 